\title{
How Many Ways Can One Draw a Graph?^
}

\author{
János Pach and Géza Tóth \\ Rényi Institute, Hungarian Academy of Sciences, Budapest, Hungary \\ Dedicated to Miklós Simonovits on his sixtieth birthday
}

\begin{abstract}
Using results from extremal graph theory, we determine the asymptotic number of string graphs with $n$ vertices, i.e., graphs that can be obtained as the intersection graph of a system of continuous arcs in the plane. The number becomes much smaller, for any fixed $d$, if we restrict our attention to systems of arcs, any two of which cross at most $d$ times. As an application, we estimate the number of different drawings of the complete graph $K_{n}$ with $n$ vertices under various side conditions.
\end{abstract}

\section{Introduction}

Given a simple graph $G$, is it possible to represent its vertices by simply connected regions in the plane so that two regions overlap if and only if the corresponding two vertices are adjacent? In other words, is $G$ isomorphic to the intersection graph of a set of simply connected regions in the plane? This deceptively simple extension of propositional logic and its generalizations are often referred to in the literature as topological inference problems [CGP98a, [CGP98b, CHK99]. They have proved to be relevant in the area of geographic information systems E93, [EF91 and in graph drawing DETT99. In spite of many efforts [K91a], K98] (and false claims [SP92], [ES93]), until very recently no algorithm was known for their solution. Two years ago, we showed PT02] that the problem is decidable. Shortly after a more elegant proof was found by Schaefer and Stefankovic SS01a, who went on proving that the question is in NP SS01b.

Since each element of a finite system of regions in the plane can be replaced by a simple continuous arc ("string") lying in its interior so that the intersection pattern of these arcs is the same as that of the original regions, it is enough to restrict our attention to string graphs, i.e., to intersection graphs of planar curves. As far as we know, these graphs were first studied in 1959 by S. Benzer [B59, who investigated the topology of genetic structures. Somewhat later they were also considered by F. W. Sinden S66 in Bell Labs, who was interested in electrical networks realizable by printed circuits. Sinden collaborated with R. L. Graham, who popularized the notion among combinatorists at a conference in Keszthely (Hungary), in 1976 G78. Soon after G. Ehrlich, S. Even, and R. E.

* János Pach has been supported by NSF grant CR-00-98246, PSC-CUNY Research Award 62450-0031 and OTKA-T-032452. Géza Tóth has been supported by OTKAT-032452 and OTKA-T-038397. 
Tarjan [EET76] studied string graphs (see also [K83] and [EPL72] for a special case). The aim of this paper is to estimate the number of different string graphs on $n$ vertices.

To formulate our main result precisely, we have to agree on the terminology. Let $G$ be a simple graph with vertex set $V(G)$ and edge set $E(G)$. A string representation of $G$ is an assignment of simple continuous arcs to the elements of $V(G)$ such that two arcs cross each other if and only if the corresponding vertices of $G$ are adjacent. Graph $G$ is a string graph if it has a string representation. We assume that any two arcs share only finitely many points and that at each common point the arcs properly cross, i.e., one arc passes from one side of the other arc to the other side. An intersection point of two arcs is called a crossing.

For any $d>0$, graph $G$ is a string graph of rank $d$ if it has a string representation with the property that any two strings have at most $d$ crossings.

A class $\mathcal{P}$ of labeled graphs, which is closed under isomorphism, is said to be a property. A property $\mathcal{P}$ is called hereditary if every induced subgraph of every member of $\mathcal{P}$ belongs to $\mathcal{P}$. Let $\mathcal{P}^{n}$ denote the set of all (labeled) graphs on the vertex set $\{1,2, \ldots, n\}$ that belong to $\mathcal{P}$. In the combinatorics literature, the function $\left|\mathcal{P}^{n}\right| \leq 2^{\left(\begin{array}{c}n \\ 2\end{array}\right)}$ is often called the speed of property $\mathcal{P}$, and there are several well known estimates on its growth rate as $n$ increases.

Let $\mathcal{S}$ and $\mathcal{S}_{d}$ denote the classes of all string graphs and all string graphs of rank $d$, respectively. Clearly, these are hereditary properties and we have $\mathcal{S}_{1} \subseteq \mathcal{S}_{2} \subseteq \cdots \subseteq \mathcal{S}$. Our first goal is to estimate their speeds.

Theorem 1. For the number $\left|\mathcal{S}^{n}\right|$ of all string graphs on $n$ labeled vertices, we have

$$
2^{\frac{3}{4}\left(\begin{array}{l}
n \\
2
\end{array}\right)} \leq\left|\mathcal{S}^{n}\right| \leq 2^{\left(\frac{3}{4}+o(1)\right)\left(\begin{array}{l}
n \\
2
\end{array}\right) .}
$$

Theorem 2. For any $d>0$, the number $\left|\mathcal{S}_{d}^{n}\right|$ of all string graphs of rank $d$ satisfies $\left|\mathcal{S}_{d}^{n}\right| \leq 2^{o\left(n^{2}\right)}$.

We do not have any better lower bound on $\left|\mathcal{S}_{d}^{n}\right|$ than $2^{\Omega(n \log n)}$, which follows from the fact that the vertex set has this many different permutations.

A drawing of a graph is a mapping $f$ which assigns to each vertex of $G$ a distinct point in the plane and to each edge $u v$ a continuous arc between $f(u)$ and $f(v)$, not passing through the image of any other vertex. For simplicity, the point assigned to a vertex is also called a vertex and an arc assigned to an edge is also called an edge of the drawing, and, if this leads to no confusion, it is also denoted by $u v$. We assume that (a) two edges have only finitely many points in common, and (b) if two edges share an interior point $p$, then they properly cross at $p$. Two drawings of $G$ are said to be essentially equivalent the set of crossing pairs of edges is the same in the two drawings. Otherwise, they are essentially different.

Let $\Delta(n)$ and $\bar{\Delta}(n)$ denote the number of essentially different drawings and essentially different straight-line drawings, resp., of the complete graph $K_{n}$ with $n$ vertices. For any $d>0$, let $\Delta_{d}(n)$ denote the number of drawings with the 
property that any two edges have at most $d$ points in common. Clearly, we have

$$
\bar{\Delta}(n) \leq \Delta_{1}(n) \leq \Delta_{2}(n) \leq \Delta_{3}(n) \leq \ldots \leq \Delta(n),
$$

for every $n$.

In Sections 2 and 3, we review the extremal graph theoretic tools used in this paper and establish Theorem 1, respectively. In Section 4 we prove Theorem 2 in the special case $d=1$. The proof in the general case is based on the same ideas, but it is technically more complicated, and it is omitted in this extended abstract. In Section 5, we deduce the following estimates.

Theorem 3. For the number of essentially different drawings of $K_{n}$ under various restrictions, we have

(i) $2^{\Omega(n \log n)} \leq \bar{\Delta}(n) \leq 2^{O(n \log n)}$;

(ii) $2^{\Omega\left(n^{2}\right)} \leq \Delta_{1}(n) \leq 2^{O\left(n^{2} \log n\right)}$;

(iii) $2^{\Omega\left(n^{2} \log n\right)} \leq \Delta_{d}(n) \leq 2^{o\left(n^{4}\right)}$, for any fixed $d \geq 2$;

(iv) $2^{\Omega\left(n^{4}\right)} \leq \Delta(n) \leq 2^{O\left(n^{4}\right)}$.

\section{Tools from Extremal Graph Theory}

One of the central questions in extremal graph theory [B78] is the following. Given a graph $H$, what is the maximum number of edges that a graph of $n$ vertices can have if it does not contain $H$ as a (not necessarily induced) subgraph? This quantity is usually denoted by $\operatorname{ex}(n, H)$.

Obviously, the property that a graph is $H$-free, is hereditary. Let $\operatorname{Forb}(n, H)$ denote the speed of this property, i.e., the number of graphs on $n$ labeled vertices that do not contain $H$ as a subgraph. It turns out that the growth rate of these functions crucially depends on the chromatic number $\chi(H)$ of $H$.

Theorem 2.1. (Erdős-Stone [ES46], Erdős-Simonovits [ES66]) For any graph $H$, we have

$$
\operatorname{ex}(n, H)=\left(1-\frac{1}{\chi(H)-1}\right) \frac{n^{2}}{2}+o\left(n^{2}\right) .
$$

Theorem 2.2. (Erdős-Frankl-Rödl EFR86]) For any graph H, we have

$$
\operatorname{Forb}(n, H)=2^{(1+o(1)) \operatorname{ex}(n, H)} .
$$

If we want to establish analogous results for graphs containing no induced subgraph isomorphic to $H$, then the first difficulty we have to face is the following: unless $H$ is a complete graph, the maximum number of edges that a graph of $n$ vertices can have without containing an induced copy of $H$ is $\left(\begin{array}{l}n \\ 2\end{array}\right)$. Thus, Theorem 2.1 does not have a direct analogue. Nevertheless, set

$$
\operatorname{ex}^{*}(n, H):=\left(1-\frac{1}{\tau(H)-1}\right) \frac{n^{2}}{2}+o\left(n^{2}\right),
$$


where the relevant quantity, $\tau(H)$, taking the place of the chromatic number is defined as follows.

We say that $H$ is $(r, s)$-colorable for some $0 \leq s \leq r$ if there is an $r$-coloring of the vertex set $V(H)$, in which the first $s$ color classes are cliques (i.e., induce complete subgraphs) and the remaining $r-s$ color classes are independent sets (i.e., induce empty subgraphs). Let $\mathcal{C}(r, s)$ denote the class of all $(r, s)$-colorable graphs, i.e.,

$$
\mathcal{C}(r, s)=\{H: H \text { is }(r, s) \text {-colorable }\} .
$$

Let $\tau(H)$ be the minimum integer $r$ such that $H$ is $(r, s)$-colorable for all $0 \leq$ $s \leq r$. Clearly, we have $\tau(H) \geq \chi(H)$, for every $H$.

Let $\operatorname{Forb}^{*}(n, H)$ stand for the number of graphs on $n$ labeled vertices which does contain $H$ as an induced subgraph.

Theorem 2.3. (Prömel-Steger [PS92]) For any graph H, we have

$$
\operatorname{Forb}^{*}(n, H)=2^{(1+o(1)) \operatorname{ex}^{*}(n, H)} \text {. }
$$

Using Szemerédi's Regularity Lemma, Bollobás and Thomason [BT97] generalized this result to any nonempty hereditary graph property $\mathcal{P}$. Define the coloring number $r(\mathcal{P})$ of $\mathcal{P}$ as the largest integer $r$ for which there is an $s$ such that all $(r, s)$-colorable graphs have property $\mathcal{P}$. That is,

$$
r(\mathcal{P})=\max \{r: \text { there exists } 0 \leq s \leq r \text { such that } \mathcal{P} \supset \mathcal{C}(r, s)\} .
$$

Consequently, for any $0 \leq s \leq r(\mathcal{P})+1$, there exists an $(r(\mathcal{P})+1, s)$-colorable graph that does not have property $\mathcal{P}$.

In the special case when $\mathcal{P}$ is the property that the graph does not contain any induced subgraph isomorphic to $H$, we have $r(\mathcal{P})=\tau(H)-1$.

Theorem 2.4. (Bollobás-Thomason BT97]) Let $\mathcal{P}$ be a nontrivial hereditary property of graphs, and let $\mathcal{P}^{n}$ denote the set of all graphs in $\mathcal{P}$ on the vertex set $\{1,2, \ldots n\}$. Then the speed of property $\mathcal{P}$ satisfies

$$
\left|\mathcal{P}^{n}\right|=2^{\left(1-\frac{1}{r(\mathcal{P})}+o(1)\right)\left(\begin{array}{l}
n \\
2
\end{array}\right)}
$$

where $r(\mathcal{P})$ is the coloring number of $\mathcal{P}$.

\section{String Graphs - Proof of Theorem 1}

We start with the lower bound. Consider four pairwise tangent non-overlapping disks $D_{i}, 1 \leq i \leq 4$, in the plane (see Fig. 1). Assume for simplicity that $n$ is divisible by 4 . The proof for other values of $n$ is analogous. Replace the boundary of each $D_{i}$ by $n / 4$ slightly smaller concentric circles $C_{i k}, 1 \leq k \leq n / 4$, running very close to it. Fix a pair $(i, j), 1 \leq i<j \leq 4$. By local deformation of every $C_{i k}$ in a small neighborhood of the point of tangency of $D_{i}$ and $D_{j}$, we can achieve that every $C_{i k}$ has a point lying outside every other $C_{i h}, h \neq k$. For 
every $1 \leq l \leq n / 4$ and for any predetermined set of indices $K_{l} \subseteq\{1,2, \ldots, n / 4\}$, we can now slightly modify $C_{j l}$ so that it would intersect a curve $C_{i k}$ if and only if $k \in K_{l}$. In other words, we can arbitrarily specify the bipartite crossing pattern between the curves $C_{i k}$ and $C_{j l}, 1 \leq k, l \leq n / 4$. Repeating the same procedure for every pair $(i, j)$, we can obtain any 4 -partite crossing pattern between the 4 classes, each containing $n / 4$ curves. Note that every $C_{i k}$ is a closed curve, but deleting any point of it which does not belong to another curve it becomes a string. Thus, the number of essentially different string graphs is at

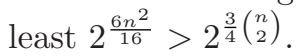

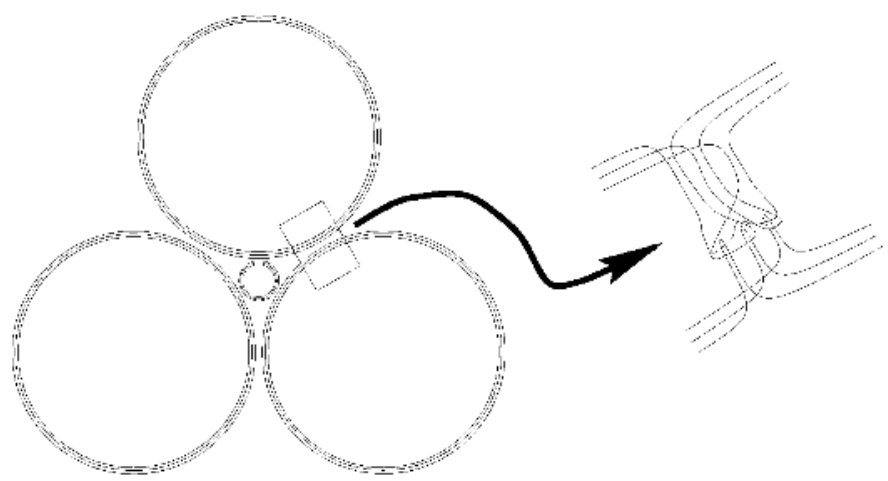

Fig. 1. Lower bound construction for the number of string graphs.

Next, we establish the upper bound. For any $r \geq 2$, let $G_{r}$ be a graph with vertex set

$$
V\left(G_{r}\right)=\left\{v_{i j}: 1 \leq i, j \leq r\right\}
$$

and edge set

$$
E\left(G_{r}\right)=\left\{v_{i j} v_{i k}: 1 \leq i, j, k \leq r, j \neq k\right\},
$$

where $v_{i j}=v_{j i}$, for every $i$ and $j$. In other words, the vertices of $G_{r}$ represent the vertices and the edges of the complete graph $K_{r}$, two vertices of $G_{r}$ being connected if the corresponding two edges of $K_{r}$ share an endpoint or the corresponding edge and vertex of $K_{r}$ are incident.

Lemma 3.1. We have $\tau\left(G_{r}\right)=r$.

Proof. The vertices $v_{1 j}, 1 \leq j \leq r$ form a clique of size $r$. Therefore, we have $\tau\left(G_{r}\right) \geq \chi\left(G_{r}\right) \geq r$.

Now we show by induction on $r$ that $\tau\left(G_{r}\right)=r$. This is true for $r=2$. Let $r>2$ be fixed and assume $\tau\left(G_{r-1}\right)=r-1$. We have to show that, for any $0 \leq s \leq r$, the vertices of $G_{r}$ can be colored by $r$ colors so that $s$ color classes induce cliques and the remaining $r-s$ color classes are independent sets. 
For $s=0$, the following coloring will satisfy the requirements. For any $1 \leq$ $k \leq r$, color a vertex $v_{i j}$ with color $k$ if and only if $i+j \equiv k \bmod r$. Clearly, each vertex of $G_{r}$ receives a color and each color class is an independent set.

If $s>0$, color each vertex of the clique $\left\{v_{1 j}: 1 \leq j \leq r\right\}$ with color 1 . The uncolored vertices induce a subgraph isomorphic to $G_{r-1}$, for which we have $\tau\left(G_{r-1}\right)=r-1$, by the induction hypothesis. So the remaining vertices can be colored by $r-1$ colors so that $s-1$ color classes induce cliques and the other $r-s$ are independent sets.

Lemma 3.2. $G_{5}$ is not a string graph. Proof. Suppose that $G_{5}$ has a string representation. Continuously contract each of string (arc) representing $v_{i i}(1 \leq$ $i \leq 5)$ to a point $p_{i}$, without changing the crossing pattern. For every pair $i \neq j$, consider the portion of the arc representing $v_{i j}$ between the points $p_{i}$ and $p_{j}$. These arcs define a drawing of $K_{5}$, in which no two independent edges cross each other. However, $K_{5}$ is not a planar graph, hence, by a well known theorem of Hanani and Tutte Ch34, T70, no such drawing exists.

Now we can complete the proof of Theorem 1. By Lemma 3.2, a string graph cannot have an induced subgraph isomorphic to $G_{5}$. Thus, in view of Lemma 3.1, Theorem 1 directly follows from Theorem 2.3:

$$
\left|\mathcal{S}^{n}\right| \leq \operatorname{Forb}_{n}^{*}\left(G_{5}\right)=2^{\left(\frac{3}{4}+o(1)\right)\left(\begin{array}{c}
n \\
2
\end{array}\right)} .
$$

\section{String Graphs of a Fixed Rank - Proof of Theorem 2}

In order to show that there are $2^{o\left(n^{2}\right)}$ string graphs of rank $d$, in view of Theorem 2.4 , it is enough to exhibit a $(2,0)$-colorable, a $(2,1)$-colorable, and a $(2,2)$ colorable graph such that none of them is a string graph of rank $d$.

Here we present the argument only in the special case $d=1$.

Let $H_{3,3}$ denote a graph with vertices $u_{i}, v_{j}$, and $w_{i j}, 1 \leq i, j \leq 3$ and edges $u_{i} w_{i j}, w_{i j} v_{j}$, for every $i$ and $j$. In other words, $H_{3,3}$ is the graph obtained from $K_{3,3}$, the complete bipartite graph with three vertices in its classes, by subdividing each of its edges by an extra vertex.

For any $k$, let $T_{k}$ denote a graph with vertices $v_{i},(1 \leq i \leq k)$ and $u_{I}$, for every $I \subseteq\{1,2, \ldots, k\}$. Let $v_{i}$ and $v_{j}$ be connected by an edge of $T_{k}$, for any $1 \leq i<j \leq k$, and let $v_{i}$ be connected to $u_{I}$ if and only if $i \in I$. Let $T_{k}^{\prime}$ denote the graph obtained from $T_{k}$ by adding the edges $u_{I} u_{J}$, for every $I \neq J$.

Clearly, $H_{3,3}$ is $(2,0)$-colorable (bipartite), $T_{k}$ is $(2,1)$-colorable, and $T_{k}^{\prime}$ is $(2,2)$-colorable, for every $k$. Therefore, if $\mathcal{P}=\mathcal{P}\left(H_{3,3}, T_{k}, T_{k}^{\prime}\right)$ denotes the property that a graph does not contain $H_{3,3}, T_{k}$, or $T_{k}^{\prime}$ as an induced subgraph, then $\mathcal{P}$ is a hereditary property with coloring number $r(\mathcal{P})=\infty$. Hence, by Theorem 2.4 , for the number of graphs on $n$ labeled vertices, satisfying property $\mathcal{P}$, we have $\left|\mathcal{P}^{n}\right|=2^{o\left(n^{2}\right)}$.

It remains to prove the following statement, which implies that $\mathcal{S}_{1}^{n} \subseteq \mathcal{P}^{n}$ if $k$ is large enough. 
Lemma 4.1. A string graph of order 1 cannot contain $H_{3,3}, T_{k}$, or $T_{k}^{\prime}$ as an induced subgraph, provided that $k$ is sufficiently large.

Proof. It is well known that a string graph cannot contain $H_{3,3}$ as an induced subgraph (see e.g. EET76,

Using the notation in the definition of $T_{k}$ (and $T_{k}^{\prime}$ ), let $v_{i}, 1 \leq i \leq k$ and $u_{I}$, $I \subseteq\{1,2, \ldots, k\}$ stand for the vertices of $T_{k}$ (and $T_{k}^{\prime}$, resp.), and suppose that $T_{k}$ (and $T_{k}^{\prime}$, resp.) has a string representation in which any two strings cross at most once. For simplicity, we use the same notation for the strings as for the corresponding vertices.

Fix arbitrarily an orientation of each string. For any triple $(x, y, z), 1 \leq x<$ $y<z \leq k$, let $f_{x y z}=1$ if along $v_{y}$ the crossing with $v_{x}$ follows the crossing with $v_{z}$. Otherwise, set $f_{x y z}=0$.

By Ramsey's theorem, there exists a "homogeneous" subset $J \subseteq\{1,2, \ldots, k\}$, $|J| \geq \log \log k$, such that $f_{x y z}$ is constant over all triples $(x, y, z), 1 \leq x<$ $y<z \leq k, x, y, z \in J$. We can assume without loss of generality that $J=$ $\{1,2, \ldots, m\}$, where $m \geq \log \log k$.

For any $1 \leq i \leq m$, the string $v_{i}$ crosses all other $v_{j}, 1 \leq j \leq m, i \neq j$ exactly once. Since $f_{x i z}$ is constant over all triples $(x, i, z), 1 \leq x<i<z \leq k$, one can find a non-crossing point on $v_{i}$ that divides $v_{i}$ into two parts, $v_{i}^{<}$and $v_{i}^{>}$, containing all crossings between $v_{i}$ and $v_{x}$ with $x<i$ and between $v_{i}$ and $v_{z}$ with $z>i$, respectively. The $\operatorname{arcs} v_{i}^{<}$and $v_{i}^{>}$are called the lower part and the upper part of $v_{i}$, respectively.

Construct two 42-uniform hypergraphs, $H^{<}$and $H^{>}$, both on the vertex set $\{1,2, \ldots, m\}$, as follows. For any $1 \leq x_{1}<x_{2}<\cdots<x_{83} \leq m$, there exists a string $u=u_{\left\{x_{1}, x_{2}, \ldots, x_{83}\right\}}$ that crosses $v_{x_{1}}, v_{x_{2}}, \ldots, v_{x_{83}}$, but no other $v_{j}$. The string $u$ crosses either the lower or the upper part of each $v_{x_{i}}$, so for at least 42 indices $1 \leq i \leq 83$ it will cross, say, the lower (resp., upper) part. Suppose, for example, that $u$ crosses the lower (resp., upper) parts of $v_{x_{1}}, v_{x_{2}}, \ldots, v_{x_{42}}$. Then add the hyperedge $\left\{x_{1}, x_{2}, \ldots, x_{42}\right\}$ to $H^{<}$(resp., to $H^{>}$).

Repeating the above procedure for every 83-tuple $1 \leq x_{1}<x_{2}<\cdots<$ $x_{83} \leq m$, the total number of hyperedges in $H^{<}$and $H^{>}$with repetitions is $\left(\begin{array}{c}m \\ 83\end{array}\right)$. However, the multiplicity of each hyperedge is at most $\left(\begin{array}{c}m-42 \\ 41\end{array}\right)$. Thus, the total number of distinct hyperedges in $H^{<}$and $H^{>}$is $\Omega\left(m^{42}\right)$ (i.e., at least constant times $\left.\mathrm{m}^{42}\right)$. Suppose without loss of generality that $H^{<}$has $\Omega\left(m^{42}\right)$ distinct hyperedges.

We can now apply a well known result of Erdős [E65 (see also B78] and PA95, p. 151) to conclude that, for any fixed $l$ and sufficiently large $m$, our hypergraph $H^{<}$contains a complete 42-partite, 42-uniform subhypergraph $K_{l, \ldots, l}^{42}$ with $l$ elements in each of its classes. (That is, $K_{l, \ldots, l}^{42}$ has $42 l$ vertices, divided into 42 classes of size $l$, and it consists of all 42-tuples that contain one vertex from each class.)

For simplicity, denote by $s_{i}^{j}, 1 \leq i \leq 42,1 \leq j \leq l$ the lower parts $v_{x_{k}}^{<}$of the strings $v_{x_{k}}$ corresponding to the vertices of $K_{l, \ldots, l}^{42}$. By the construction, for each 42-tuple $\left(j_{1}, \ldots, j_{42}\right), 1 \leq j_{1}, \ldots, j_{42} \leq l$, there exists a string $u_{j_{1}, \ldots, j_{42}}$ that crosses $s_{1}^{j_{1}}, \ldots, s_{42}^{j_{42}}$, but no other string $s_{i}^{j}$. 


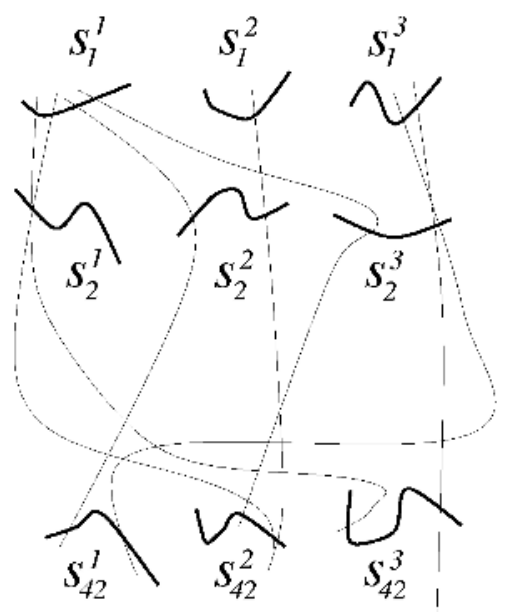

Fig. 2. Some of the strings representing a $K_{3, \ldots, 3}^{42}$.

Color the 42 -tuples $\left(j_{1}, \ldots, j_{42}\right)$ with 42 ! colors, according to order in which the crossings with $s_{1}^{j_{1}}, \ldots, s_{42}^{j_{42}}$ occur along $u_{j_{1}, \ldots, j_{42}}$. Thus, we can find at least $\Omega\left(l^{42}\right)$ 42-tuples of the same color (say, white). Suppose without loss of generality that, for each such 42 -tuple $\left(j_{1}, \ldots, j_{42}\right)$, the string $u_{j_{1}, \ldots, j_{42}}$ first crosses $s_{1}^{j_{1}}$, then $s_{2}^{j_{2}}, \ldots$, and finally $s_{42}^{j_{42}}$. Applying Erdős's result again, if $l$ is sufficiently large, we can find a subhypergraph $K_{3, \ldots, 3}^{42} \subset K_{l, \ldots, l}^{42}$, all of whose 42-tuples are white. Again, we can assume without loss of generality that the strings corresponding to the vertices of $K_{3, \ldots, 3}^{42}$ are $s_{i}^{j}, 1 \leq i \leq 42,1 \leq j \leq 3$. Recall that each $s_{i}^{j}$ is the lower part of an original string $v_{x}$, therefore, no two $s_{i}^{j}$ can cross each other. (Indeed, the intersection of $v_{x}$ and $v_{y}, x<y$, must belong to the upper part of $v_{x}$ and at to the lower part of $v_{y}$.)

Summarizing: we have $3 \cdot 42=126$ strings $s_{i}^{j}, 1 \leq i \leq 42,1 \leq j \leq 3$, no two of which intersect. Moreover, for each 42-tuple $\left(j_{1}, \ldots, j_{42}\right), 1 \leq j_{1}, \ldots, j_{42} \leq 3$, there is a string $u_{j_{1}, \ldots, j_{42}}$ that intersects the strings $s_{1}^{j_{1}}, \ldots, s_{42}^{j_{42}}$ in this order, and does not intersect any other $s_{i}^{j}$. (See Fig. 2.) We would like to show that there are two different strings of the type $u_{j_{1}, \ldots, j_{42}}$ that cross more than once. First, we give a lower bound for the number of crossings $\mathrm{CR}(u, u)$ between strings of type $u_{j_{1}, \ldots, j_{42}}$.

Let $1 \leq x \leq 41$ be fixed. For any pair $y, z, 1 \leq y, z \leq 3$, consider all strings $u_{j_{1}, \ldots, j_{42}}$ with $j_{x}=y$ and $j_{x+1}=z$, and let $\Gamma_{y, z}$ denote the set of their portions between their intersections with $s_{x}^{y}$ and $s_{x+1}^{z}$. Clearly, we have $\left|\Gamma_{y, z}\right|=3^{40}$. Pick one element from each $\Gamma_{y, z}, 1 \leq y, z \leq 3$, and notice that at least one pair among these 9 arcs must be crossing, otherwise, together with the strings $s_{x}^{1}, s_{x}^{2}, s_{x}^{3}, s_{x+1}^{1}, s_{x+1}^{2}, s_{x+1}^{3}$, they would give a string representation of $H_{3,3}$, which is impossible (see the first paragraph of this proof). Thus, for a fixed $x$, the total number of crossings between the elements of $\Gamma_{y, z}$ and $\Gamma_{y^{\prime}, z^{\prime}}$ over all $y, z, y^{\prime}, z^{\prime}$, 
$1 \leq y, z, y^{\prime}, z^{\prime} \leq 3,(y, z) \neq\left(y^{\prime}, z^{\prime}\right)$ is at least

$$
\frac{\prod_{1 \leq y, z \leq 3}\left|\Gamma_{y, z}\right|}{3^{7 \cdot 40}}=\frac{3^{9 \cdot 40}}{3^{7 \cdot 40}}=3^{80} .
$$

Here the denominator, $3^{7 \cdot 40}$, is the number of 9-tuples of arcs, one from each set $\Gamma_{y, z}, 1 \leq y, z \leq 3$, in which a crossing pair of arcs is fixed. Repeating this count for every $x, 1 \leq x \leq 41$ and noticing that every time we count different crossings, we obtain that

$$
\mathrm{CR}(u, u) \geq 41 \cdot 3^{80}
$$

On the other hand, the number of strings of type $u_{j_{1}, \ldots, j_{42}}$ is $3^{42}$. If any two of them cross at most once, than $\mathrm{CR}(u, u)<3^{84} / 2$, which is a contradicts the above inequality. This completes the proof of the lemma.

\section{Drawings of Complete Graphs - Proof of Theorem 3}

(i) It is easy to see that the order type on the vertices of $K_{n}$ (i.e., the orientation of its triples) determines the set of crossing pairs of edges, So the upper bound follows from a result of Goodman and Pollack [GP86], that there are at most $n^{6 n}$ different order types on $n$ points. On the other hand, we can place the vertices of $K_{n}$ on a circle, in $(n-1)$ ! different cyclic order, and each placement gives a different list of crossing pairs of edges. It is also easy to come up with a list of $n^{\Omega(n)}$ drawings such that by relabelling the vertices of any one of them, we do not obtain a drawing essentially the same as another.

(ii) Suppose $n$ is divisible by 4 , and let $v_{i}=(-1, i), u_{j}=(1, j)$, and $w_{k}=$ $(0, k / 2)$, for any $1 \leq i, j \leq n / 4$ and $1 \leq k \leq n / 2$. For every $1 \leq k<n / 2$, connect $w_{k}$ and $w_{k+1}$ by a straight-line segment. Furthermore, connect every $v_{i}$ to every $u_{j}$ by a line segment so that each such segment passes through some point $w_{k}$. By slightly bending each edge $v_{i} u_{j}$, but keeping its endpoints fixed, we can achieve that it passes either slightly above or slightly below $w_{i+j}$. At each edge $v_{i} u_{j}$, we have two choices, so there are $2^{n^{2} / 16}$ possibilities. In each drawing, any two edges cross at most once, and different choices give rise to different crossing patterns. (Indeed, $v_{i} u_{j}$ passes above $w_{i+j}$ if and only if it crosses the edge $w_{i+j} w_{i+j+1}$.) Finally, one can slightly perturb the vertices so that no three of them would be collinear, and connect the missing pairs by straight-line segments without creating more than one crossing between any pair of edges. Therefore, the number of different crossing patterns is at least $2^{n^{2} / 16}$.

As for the upper bound, for a fixed drawing, for each vertex $v_{i}$, list the edges incident to $v_{i}$ in clockwise order around $v_{i}$. For every vertex, we have $(n-2)$ ! possibilities, so there are $((n-2) !)^{n}<2^{n^{2} \log n}$ different sets of lists. We claim that this set of lists uniquely determines the crossing pattern. To see this take two edges, $v_{1} v_{2}$ and $v_{3} v_{4}$, and consider the drawing of $K_{4}$ induced by these vertices, as a drawing on the sphere. Two spherical drawings of $K_{4}$ are combinatorially 

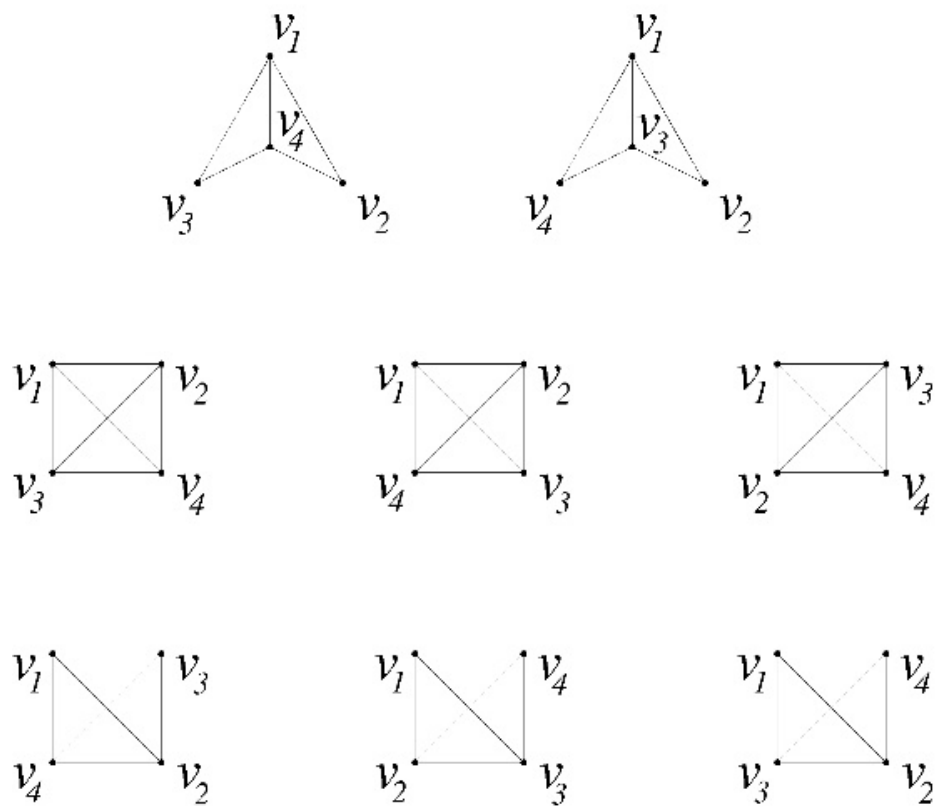

Fig. 3. The eight combinatorially different drawings of $K_{4}$.

equivalent if the corresponding maps are isomorphic. There are 8 combinatorially different drawings of $K_{4}$, with the property that any two edges have at most one point in common (see Fig. 3), and these drawings can be distinguished by looking at the cyclic orders of edges incident to a vertex. Hence, the cyclic order of edges at the vertices determines whether $v_{1} v_{2}$ and $v_{3} v_{4}$ cross each other.

(iii) Suppose $n$ is divisible by 3 . For $i=1,2, \ldots, n / 3$, let $v_{i}=(-1, i), w_{i}=(0, i)$, and $u_{i}=(1, i)$. Connect every $v_{i}$ to every $u_{j}$, as follows. Choose a number $k$, $0 \leq k<n / 3$, and connect both $v_{i}$ and $u_{j}$ to $(0, k+\varepsilon)$ by a segment. Also connect any two consecutive $w_{i}$ 's by a segment. In the resulting drawing, any two have at most two common points, and a different choice for any $v_{i} u_{j}$ results a different crossing pattern. Therefore, the number of different crossing patterns is $n / 3^{n^{2} / 9}$. Clearly, each of these drawings can be extended to a drawing of the complete graph such that still any two have at most two common points. For instance, slightly perturb the points together with the existing edges, so that the points are in general position, and add the missing edges as segments.

For the upper bound, apply Theorem 2 for the edges of $K_{n}$ regarded as $\left(\begin{array}{l}n \\ 2\end{array}\right)$ strings.

(iv) Suppose $n$ is even, and let $v_{i}=(-1, i), u_{i}=(1, i)$, for $1 \leq i \leq n / 2$. For any $i, j, 1 \leq i<j \leq n / 2$, connect $v_{i}$ with $(0, n i+j)$ and connect $(0, n i+j)$ with $v_{j}$. Now, all vertices $v_{i}$ and all edges connecting them are on the left side of the line $x=0$ such that each of the edges has exactly one point on that line, and all these points are different. On the other hand, all vertices $u_{i}$, are on the 
right-hand side of the line $x=0$. So, for any $p, q, 1 \leq p<q \leq n / 2$, and for any set $K_{p q} \subseteq\{(i, j): 1 \leq i<j \leq n / 2\}$, we can draw the edge $v_{p} v_{q}$ so that it crosses $u_{i} u_{j}(i<j)$ if and only if $(i, j) \in K_{p q}$ (cf. proof of Theorem 1).

\section{References}

[B59] S. Benzer, On the topology of the genetic fine structure, Proceedings of the National Academy of Sciences of the United States of America 45 (1959), 1607-1620.

[B78] B. Bollobás, Extremal Graph Theory, London Mathematical Society Monographs 11, Academic Press. [Harcourt Brace Jovanovich, Publishers], London-New York, 1978.

[BT97] B. Bollobás and A. Thomason, Hereditary and monotone properties of graphs, in: The mathematics of Paul Erdös II, (R. L. Graham and J. Nešetřil, eds.) Algorithms Combin. 14, Springer, Berlin, 1997, 70-78.

[CGP98a] Z.-Z. Chen, M. Grigni, and C. H. Papadimitriou, Planar map graphs, in: STOC' '98, ACM, 1998, 514-523.

[CGP98b] Z.-Z. Chen, M. Grigni, and C. H. Papadimitriou, Planar topological inference, (Japanese) in: Algorithms and Theory of Computing (Kyoto, 1998) Sūrikaisekikenkyūsho Kōkyūroku 1041 (1998), 1-8.

[CHK99] Z.-Z. Chen, X. He, and M.-Y. Kao, Nonplanar topological inference and political-map graphs, in: Proceedings of the Tenth Annual ACM-SIAM Symposium on Discrete Algorithms (Baltimore, MD, 1999), ACM, New York, 1999, 195-204.

[Ch34] Ch. Chojnacki (A. Hanani), Über wesentlich unplättbare Kurven im dreidimensionalen Raume, Fund. Math. 23 (1934), 135-142.

[DETT99] G. Di Battista, P. Eades, R. Tamassia, and I. G. Tollis, Graph Drawing, Prentice Hall, Upper Saddle River, NJ, 1999.

[E93] M. Egenhofer, A model for detailed binary topological relationships, Geomatica 47 (1993), 261-273.

[E65] P. Erdős, On some extremal problems in extremal graph theory, Israel J. Math. 3 (1965), 113-116.

[EF91] M. Egenhofer and R. Franzosa, Point-set topological spatial relations, International Journal of Geographical Information Systems 5 (1991), 161-174.

[ES93] M. Egenhofer and J. Sharma, Assessing the consistency of complete and incomplete topological information, Geographical Systems 1 (1993), 47-68.

[EET76] G. Ehrlich, S. Even, and R. E. Tarjan, Intersection graphs of curves in the plane, Journal of Combinatorial Theory, Series B 21 (1976), 8-20.

[EFR86] P. Erdős, P. Frankl, and V. Rödl, The asymptotic number of graphs not containing a fixed subgraph and a problem for hypergraphs having no exponent, Graphs and Combinatorics 2 (1986), 113-121.

[ES66] P. Erdős and M. Simonovits, A limit theorem in graph theory, Studia Sci. Math. Hungar. 1 (1966), 51-57.

[ES46] P. Erdős and A. H. Stone, On the structure of linear graphs, Bulletin Amer. Math. Soc. 52 (1946), 1087-1091.

[EPL72] S. Even, A. Pnueli, and A. Lempel, Permutation graphs and transitive graphs, Journal of Association for Computing Machinery 19 (1972), 400411. 
[G80] M. C. Golumbic, Algorithmic Graph Theory and Perfect Graphs, Academic Press, New York, 1980.

[GP86] J. E. Goodman and R. Pollack, Upper bounds for configurations and polytopes in $R^{d}$, Discrete Comput. Geom. 1 (1986), 219-227.

[G78] R. L. Graham, Problem, in: Combinatorics, Vol. II (A. Hajnal and V. T. Sós, eds.), North-Holland Publishing Company, Amsterdam, 1978, 1195.

[HT74] J. Hopcroft and R. E. Tarjan, Efficient planarity testing, J. ACM 21 (1974), 549-568.

[K83] J. Kratochvíl, String graphs, in: Graphs and Other Combinatorial Topics (Prague, 1982), Teubner-Texte Math. 59, Teubner, Leipzig, 1983, 168-172.

[K91a] J. Kratochvíl, String graphs I: The number of critical nonstring graphs is infinite, Journal of Combinatorial Theory, Series B 52 (1991), 53-66.

[K91b] J. Kratochvíl, String graphs II: Recognizing string graphs is NP-hard, Journal of Combinatorial Theory, Series B $\mathbf{5 2}$ (1991), 67-78.

[K98] J. Kratochvíl, Crossing number of abstract topological graphs, in: Graph drawing (Montreal, QC, 1998), Lecture Notes in Comput. Sci. 1547, Springer, Berlin, 1998, 238-245.

[PA95] J. Pach and P. K. Agarwal, Combinatorial Geometry, Wiley Interscience, New York, 1995.

[PS01] J. Pach and J. Solymosi, Crossing patterns of segments, Journal of Combinatorial Theory, Ser. A, 96 (2001), 316-325.

[PT02] J. Pach and G. Tóth, Recognizing string graphs is decidable, Lecture Notes in Computer Science 2265, Spinger-Verlag, Berlin, 2001, 247-260. Also in: Discrete and Computational Geometry 28 (2002), 593-606.

[PS92] H. J. Prömel and A. Steger, Excluding induced subgraphs III: A general asymptotic, Random Structures and Algorithms 3 (1992), 19-31.

[SS01a] M. Schaefer and D. Stefankovič, Decidability of string graphs, Proceedings of the 33rd Annual Symposium on the Theory of Computing, 2001, 241-246.

[SS01b] M. Schaefer, E. Sedgwick and D. Stefankovič, Recognizing string graphs in NP, Proceedings of the 34 rd Annual Symposium on the Theory of Computing, 2002, 1-6.

[S66] F. W. Sinden, Topology of thin film RC circuits, Bell System Technological Journal (1966), 1639-1662.

[SP92] T. R. Smith and K. K. Park, Algebraic approach to spatial reasoning, International Journal of Geographical Information Systems 6 (1992), 177-192.

[T70] W. T. Tutte, Toward a theory of crossing numbers, J. Combinatorial Theory 8 (1970), 45-53. 